\title{
Ultrasonic waves in uniaxially stressed multilayered and one-dimensional phononic structures: Guided and Floquet wave analysis
}

Andriejus Demčenko, Rab Wilson, Jonathan M. Cooper, Michael Mazilu, and Arno W. F. Volker

Citation: The Journal of the Acoustical Society of America 144, 81 (2018); doi: 10.1121/1.5044528

View online: https://doi.org/10.1121/1.5044528

View Table of Contents: http://asa.scitation.org/toc/jas/144/1

Published by the Acoustical Society of America

\section{Articles you may be interested in}

Finite-element analysis of non-collinear mixing of two lowest-order antisymmetric Rayleigh-Lamb waves

The Journal of the Acoustical Society of America 144, 53 (2018); 10.1121/1.5044422

On the frequency domain formulation of the generalized sound extrapolation method

The Journal of the Acoustical Society of America 144, 24 (2018); 10.1121/1.5044515

Modeling of the multimodal radiation from an open-ended waveguide

The Journal of the Acoustical Society of America 143, 3520 (2018); 10.1121/1.5041268

Estimating the Green's function using a single channel dual-beam interferometer

The Journal of the Acoustical Society of America 144, 124 (2018); 10.1121/1.5045329

Ultrasound-based cell sorting with microbubbles: A feasibility study

The Journal of the Acoustical Society of America 144, 41 (2018); 10.1121/1.5044405

Horizontal directivity patterns differ between vowels extracted from running speech

The Journal of the Acoustical Society of America 144, EL7 (2018); 10.1121/1.5044508 


\title{
Ultrasonic waves in uniaxially stressed multilayered and one-dimensional phononic structures: Guided and Floquet wave analysis
}

\author{
Andriejus Demčenko, ${ }^{\text {a) }}$ Rab Wilson, and Jonathan M. Cooper \\ Division of Biomedical Engineering, School of Engineering, Rankine Building, Oakfield Avenue, \\ The University of Glasgow, Glasgow G12 8LT, United Kingdom \\ Michael Mazilu \\ SUPA, School of Physics and Astronomy, University of St Andrews, St Andrews KY16 9SS, United Kingdom
}

\author{
Arno W. F. Volker \\ Acoustics and Sonar, TNO, Oude Waalsdorperweg 63, 2597 AK Den Haag, the Netherlands
}

(Received 17 April 2018; revised 11 June 2018; accepted 13 June 2018; published online 5 July 2018)

\begin{abstract}
This paper shows that acoustoelasticity in one-dimensional (1D) multilayered isotropic hyperelastic materials can be understood through the analysis of elastic wave velocities as a function of applied stress. This theoretical framework is used for eigenvalue analyses in stressed elastic structures through a reformulation of the stiffness matrix method, obtaining modal solutions, as well as reflection and transmission coefficients for different multilayered configurations. Floquet wave analysis for the stressed 1D structures is supported using numerical results. (C) 2018 Author(s). All article content, except where otherwise noted, is licensed under a Creative Commons Attribution (CC BY) license (http://creativecommons.org/licenses/by/4.0/). https://doi.org/10.1121/1.5044528
\end{abstract}

Pages: 81-91

\section{INTRODUCTION}

Multilayered elastic structures are widely investigated in a broad range of fields including geophysics, bioengineering, manufacturing and communications. In these studies, one can readily characterise both naturally layered structures, such as soil and skin, and artificial structures including material composites. Despite their apparent differences in composition, these layered media can, in principle, be analyzed in terms of their interaction with elastic guided waves, such as Lamb or surface waves.

In general, the propagation velocity of such acoustic waves will change in the presence of static or residual stresses within the layered media (a well understood phenomena known as the acoustoelastic effect) (Hughes and Kelly, 1953; Pao and Gamer, 1985). Such changes in propagation can be used in a variety of applications including the inversion of media properties (Korneev and Glubokovskikh, 2013), the detection of soft tissue changes (Gennisson et al., 2007) and the estimation of residual stresses in engineered structures (Hughes and Kelly, 1953; Kino et al., 1980). This effect can also be used for the measurement of the third order elastic constants (Egle and Bray, 1976) and for more accurate design of communication devices (Zhang et al., 2013) where any residual stress can affect wave propagation significantly.

Acoustoelasticity, based upon a continuum theory of small disturbances superimposed on an elastically deformed body involving third order elastic constants in the constitutive equations, was initially developed for ultrasonic bulk waves (Hughes and Kelly, 1953; Pao and Gamer, 1985). Generally,

\footnotetext{
${ }^{a)}$ Electronic mail: andriejus.demcenko@glasgow.ac.uk
}

previously reported work presents the application of the acoustoelastic theory for the analysis of guided waves (Lamb wave type) propagating in biaxially stressed plates (Gandhi et al., 2012), providing a formulation of a transfer matrix method for a single layer to calculate phase velocity dispersion curves of guided waves. Other work (Dubuc et al., 2017; Gandhi et al., 2012; Kubrusly et al., 2016; Pei and Bond, 2016) also considers Lamb wave propagation in single layered plates. Recently it has been shown that acoustoelastic theory can be understood within a framework of non-linear wave mixing (Demčenko et al., 2018).

Although acoustoelastic theory has been used to understand Lamb wave propagation in single plates, the analytical methods currently used have constraints; for example, the transfer matrix methods are numerically unstable (Rokhlin and Wang, 2002; Tan, 2006). There is also limited reported work dedicated to the acoustoelastic analysis of the guided wave in layered or multilayered plate-like structures (Mseddi et al., 2014; Osetrov et al., 2000; Qu and Liu, 1998). Two reported approaches using transfer matrix methods and recursive stiffness matrix methods (Mseddi et al., 2014; Osetrov et al., 2000) considered the acoustoelastic surface wave analysis and used phase velocity to calculate the surface wave dispersion curves although guided waves, such as Lamb waves, were not analyzed. For example, work by Qu and Liu (1998) considers acoustoelastic phase velocity Lamb wave analysis in a tri-layered structure using the transfer matrix method, although the analysis was limited to the direction of the Lamb waves propagation with respect to the direction of the applied stress, and as such, no shear horizontal wave motion coupling to sagittal wave motion was discussed in the work.

More recent research (Galich et al., 2017) presents an analysis of elastic wave propagation in finitely deformed 
layered materials within long wavelength regimes, with small amplitude motions, normal to the wave motion. However, the work did not present an analysis of the ultrasonic wave response from submersed stressed plates in terms of the reflection and transmission coefficients of ultrasonic waves.

Given the importance of such submersed and air-coupled ultrasonic measurements in both engineering research and industrial applications, including their role as multilayered composite laminates (Demčenko et al., 2006; Lee and Soutis, 2007; Li et al., 2017), it is useful to analyse such periodic structures. These composite laminates also respond mechanically to ultrasound in a manner analogous to a one-dimensional (1D) phononic structure and can be considered as having band-gaps (Kushwaha et al., 1993), enabling the use of Floquet wave theory in their analysis (Braga and Herrmann, 1992; Wang and Rokhlin, 2002a). Wave propagation analysis in more complex periodic structures has been reported for low frequency mechanical waves showing the effect of the pre-stress on the resonant bandgap position and magnitude (Wang et al., 2014).

The main aim of this work is to investigate the application of the Floquet wave theory on statically stressed multilayered 1D periodic structures to enable the development of an efficient matrix method for analysis of the elastic wave velocities with associated reflection and transmission coefficients. The analysis was conducted by means of acoustoelasticity theory for isotropic hyperelastic structures with application of this theory to stable formulation of recursive matrix methods used in the eigenvalue analysis of statically stressed elastic structures. We show that it is possible to use singular value decomposition for calculations of Floquet wave polarization vectors.

Our work first shows how the recursive matrix method, based on the stiffness matrix, can be used for guided waves analysis in stressed multilayered structures, generating modal solutions as well as the reflection and transmission coefficients. Subsequently, statically stressed periodic media were analyzed in terms of Floquet wave theory-with Floquet wavenumbers and reflection coefficients from a periodic semi-space being calculated using the recursive stiffness matrix method when periodic layered semi-space is an effective homogeneous and inhomogeneous medium. Finally, we draw general conclusions from the study in the context of its application in the analysis of composite laminates in engineering research.

\section{STIFFNESS MATRIX METHOD FOR STATICALLY STRESSED LAYERED MEDIA}

Drawing upon the stability and universality of the recursive stiffness methods in elastic wave analysis of multilayered anisotropic media (Rokhlin and Wang, 2002), we applied this method for wave propagation analysis. An idealized multilayered structure and coordinate system was used, shown in Fig. 1 where wave propagation is considered in the $x-z$ plane. The displacement vector $\mathbf{u}_{j}$ is represented as a sum of six partial waves (quasi-longitudinal, fast quasi-shear, and slow quasishear) in the $j$ th anisotropic elastic layer, accordingly

$$
\begin{aligned}
\mathbf{u}_{j}= & \sum_{n=1}^{3}\left(a_{n}^{+} \mathbf{p}_{n}^{+} e^{\mathrm{i} k_{z}^{+n}\left(z-z_{j}\right)}+a_{n}^{-} \mathbf{p}_{\mathbf{n}}^{-} e^{\mathrm{i} k_{z}^{-n}\left(z-z_{j-1}\right)}\right)_{j} \\
& \times e^{\mathrm{i}\left(k_{x} x+k_{y} y-\omega t\right)}
\end{aligned}
$$

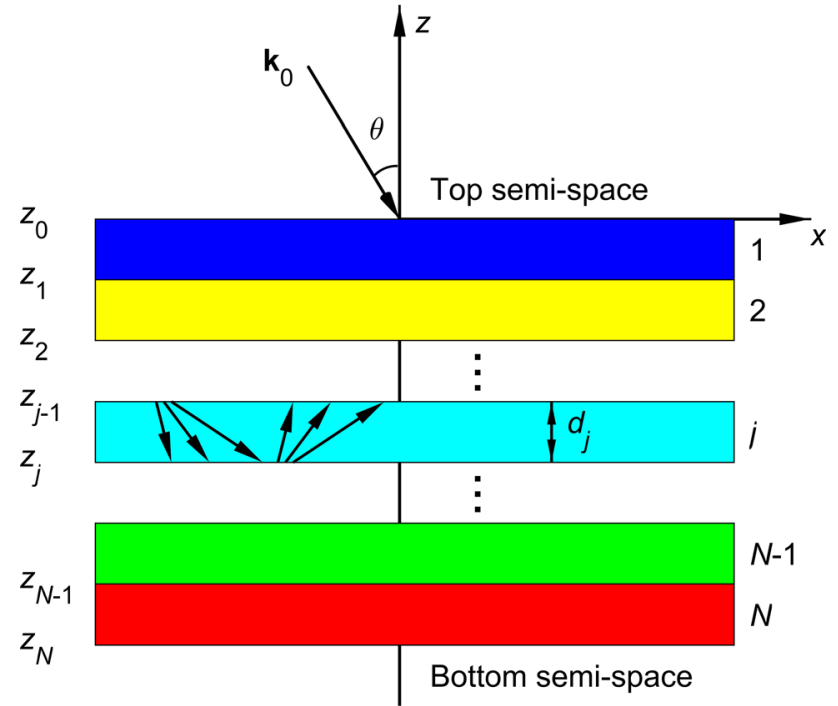

FIG. 1. (Color online) Multilayered structure and coordinate system for waves propagating in the $x-z$ plane. $\mathbf{k}_{0}$ is the incident plane wave, $\theta$ is the incidence angle of the incident wave, $d_{j}$ is the thickness of the $j$ th layer. Arrows indicate three partial waves going down and up in the $j$ th layer, respectively.

where $\mathbf{u}_{j}=\left(u_{x}^{j}, u_{y}^{j}, u_{z}^{j}\right)^{\mathrm{T}}, \mathrm{T}$ is the transpose. Further, $\mathbf{p}_{n}^{ \pm}$ $=\left(p_{x}^{ \pm}, p_{y}^{ \pm}, p_{z}^{ \pm}\right)^{\mathrm{T}}$ are the unit displacement polarization vectors corresponding to waves with $k_{z}^{ \pm}$wave vectors, respectively; \pm indicates the wave propagation direction regarding to $z$-axis, $n(=1,2,3)$ denotes the $n$th partial wave. The coordinate system is selected so that the $x-z$ plane coincides with the wave incident plane; hence $k_{y}=0$ in Eq. (1).

In the presence of static stress, the Christoffel equation for a single layer is given by (Gandhi et al., 2012)

$$
\left(A_{i j k l} k_{j} k_{l}-\rho \omega^{2} \delta_{i k}\right) p_{k}=0 .
$$

Ignoring rotation terms, the nonsymmetric tensor $A_{i j k l}$ then becomes

$$
\begin{aligned}
A_{i j k l}= & c_{i j k l}+c_{j l m n} e_{m n}^{i} \delta_{i k}+c_{i j m l} e_{k m}^{i} \\
& +c_{m j k l} e_{i m}^{i}+c_{i j k l m n} e_{m n}^{i},
\end{aligned}
$$

where $k_{j, l}$ is the wave vector, $\rho$ is the volumetric mass density of the predeformed state and $\rho=\rho_{0}\left(1-e_{n n}^{i}\right)$, and $\rho_{0}$ is the volumetric mass density of the un-deformed state (we neglect density variations). $\omega=2 \pi f, f$ is the frequency, $\delta_{i k}$ is the Kronecker delta, $p_{k}$ is the unit displacement polarization. Here and below, free indexes mean summation, $c_{i j k l}$ and $c_{i j k l m n}$ are the second and third order elastic constants, respectively, and $e_{m n}^{i}$ is the incremental strain which can be calculated from the incremental stress equation (Gandhi et al., 2012)

$$
T_{i j}^{i}=c_{i j k l} e_{k l}^{i}
$$

Considering elastic wave propagation in the $x-z$ plane, Eq. (2) can be rewritten and solved for the eigenvectors $k_{z}^{ \pm n}$, 


$$
\operatorname{det}\left[\begin{array}{lll}
K_{11} & K_{12} & K_{13} \\
K_{12} & K_{22} & K_{23} \\
K_{13} & K_{23} & K_{33}
\end{array}\right]=0
$$

where the elements $K_{i k}$ in the $x-z$ plane are

$$
K_{i k}=A_{i 1 k 1} k_{x}^{2}+\left(A_{i 1 k 3}+A_{i 3 k 1}\right) k_{x} k_{z}+A_{i 3 k 3} k_{z}^{2}-\rho \omega^{2} \delta_{i k},
$$

where $k_{x}$ is the horizontal component of the incident wave $k_{0}$.

Equation (5) can be solved using Cardano's method for solving cubic equations (Mignogna, 1989). When the vertical wavenumbers $k_{z}$ are known, the unit displacement polarization vectors $p_{n}^{ \pm}$are calculated as eigenvectors of Eq. (5). A displacement polarization calculation procedure based upon the adjugate tensor for Cristoffel's tensor is given in Rokhlin et al. (1986), and Rokhlin and Wang (2002) and it is different from the procedure used in Gandhi et al. (2012).

Normal power flux was used to sort the wavenumbers due to its applicability to Floquet wavenumbers (Potel et al., 2001) with $k_{z}$ arranged according to propagation directions (up $z^{+}$and down $z^{-}$).

In the presence of the static stress, the stress vector of the partial waves is given as

$$
\begin{aligned}
\boldsymbol{\sigma}_{j}= & \sum_{n=1}^{3}\left(a_{n}^{+} \mathbf{d}_{\mathbf{n}}^{+} e^{\mathrm{i} k_{z}^{+n}\left(z-z_{j}\right)}+a_{n}^{-} \mathbf{d}_{n}^{-} e^{\mathrm{i} k_{z}^{-n}\left(z-z_{j-1}\right)}\right)_{j} \\
& \times e^{\mathrm{i}\left(k_{x} x-\omega t\right)}
\end{aligned}
$$

where $\left(d_{i}^{ \pm}\right)_{j}=\left(B_{i 3 l n} k_{n} p_{l}^{ \pm}\right)_{j}$ and (Gandhi et al., 2012)

$$
B_{i j k l}=c_{i j k l}+c_{i j m l} e_{k m}^{i}+c_{i j k l m n} e_{m n}^{i} .
$$

Single layer stiffness matrix is (Rokhlin and Wang, 2002)

$$
\begin{aligned}
{\left[\begin{array}{c}
\boldsymbol{\sigma}_{j-1} \\
\boldsymbol{\sigma}_{j}
\end{array}\right]=} & {\left[\begin{array}{cc}
\mathbf{D}^{-} & \mathbf{D}^{+} \mathbf{E}^{+} \\
\mathbf{D}^{-} \mathbf{E}^{-} & \mathbf{D}^{+}
\end{array}\right] } \\
& \times\left[\begin{array}{cc}
\mathbf{P}^{-} & \mathbf{P}^{+} \mathbf{E}^{+} \\
\mathbf{P}^{-} \mathbf{E}^{-} & \mathbf{P}^{+}
\end{array}\right]_{j}^{-1}\left[\begin{array}{c}
\mathbf{u}_{j-1} \\
\mathbf{u}_{j}
\end{array}\right],
\end{aligned}
$$

or in a compact form

$$
\left[\begin{array}{c}
\boldsymbol{\sigma}_{j-1} \\
\boldsymbol{\sigma}_{j}
\end{array}\right]=\mathbf{K}^{j}\left[\begin{array}{c}
\mathbf{u}_{j-1} \\
\mathbf{u}_{j}
\end{array}\right]=\left[\begin{array}{ll}
\mathbf{K}_{11}^{j} & \mathbf{K}_{12}^{j} \\
\mathbf{K}_{21}^{j} & \mathbf{K}_{22}^{j}
\end{array}\right]\left[\begin{array}{c}
\mathbf{u}_{j-1} \\
\mathbf{u}_{j}
\end{array}\right],
$$

where $\mathbf{P}^{ \pm}=\left[\mathbf{p}_{1}^{ \pm}, \mathbf{p}_{2}^{ \pm}, \mathbf{p}_{2}^{ \pm}\right], \mathbf{D}^{ \pm}=\left[\mathbf{d}_{1}^{ \pm}, \mathbf{d}_{2}^{ \pm}, \mathbf{d}_{2}^{ \pm}\right], \mathbf{E}^{ \pm}=\operatorname{Diag}\left[e^{\mathrm{i} k_{2}^{ \pm 1}} d_{j}\right.$, $\left.e^{\mathrm{i} k_{z}^{ \pm 2} d_{j}}, e^{\mathrm{i} k_{z}^{ \pm 3} d_{j}}\right]$.

The terms $\mathbf{P}^{ \pm}, \mathbf{D}^{ \pm}, \mathbf{E}^{ \pm}$can however be re-arranged, formulating a hybrid matrix for a layer (Tan, 2006)

$$
\begin{aligned}
{\left[\begin{array}{c}
\mathbf{u}_{j} \\
\boldsymbol{\sigma}_{j-1}
\end{array}\right]=} & {\left[\begin{array}{cc}
\mathbf{P}^{+} & \mathbf{P}^{-} \mathbf{E}^{-} \\
\mathbf{D}^{+} \mathbf{E}^{+} & \mathbf{D}^{-}
\end{array}\right] } \\
& \times\left[\begin{array}{cc}
\mathbf{D}^{+} & \mathbf{D}^{-} \mathbf{E}^{-} \\
\mathbf{P}^{+} \mathbf{E}^{+} & \mathbf{P}^{-}
\end{array}\right]_{j}^{-1}\left[\begin{array}{c}
\boldsymbol{\sigma}_{j} \\
\mathbf{u}_{j-1}
\end{array}\right],
\end{aligned}
$$

or in a compact form

$$
\left[\begin{array}{c}
\mathbf{u}_{j} \\
\boldsymbol{\sigma}_{j-1}
\end{array}\right]=\mathbf{H}^{j}\left[\begin{array}{c}
\boldsymbol{\sigma}_{j} \\
\mathbf{u}_{j-1}
\end{array}\right]=\left[\begin{array}{ll}
\mathbf{H}_{11}^{j} & \mathbf{H}_{12}^{j} \\
\mathbf{H}_{21}^{j} & \mathbf{H}_{22}^{j}
\end{array}\right]\left[\begin{array}{c}
\boldsymbol{\sigma}_{j} \\
\mathbf{u}_{j-1}
\end{array}\right] .
$$

Accordingly, the following relationships can be written for the stiffness and hybrid matrices, demonstrating how it is easy to move from one matrix system to another (Tan, 2006),

$$
\begin{aligned}
\mathbf{K}^{j} & =\left[\begin{array}{cc}
\left(\mathbf{H}_{11}^{j}\right)^{-1} & \left(\mathbf{H}_{11}^{j}\right)^{-1} \mathbf{H}_{12}^{j} \\
\mathbf{H}_{21}^{j}\left(\mathbf{H}_{11}^{j}\right)^{-1} & \mathbf{H}_{22}^{j}-\mathbf{H}_{21}^{j}\left(\mathbf{H}_{11}^{j}\right)^{-1} \mathbf{H}_{12}^{j}
\end{array}\right], \\
\mathbf{H}^{j} & =\left[\begin{array}{cc}
\left(\mathbf{K}_{11}^{j}\right)^{-1} & -\left(\mathbf{K}_{11}^{j}\right)^{-1} \mathbf{K}_{12}^{j} \\
\mathbf{K}_{21}^{j}\left(\mathbf{K}_{11}^{j}\right)^{-1} & \mathbf{K}_{22}^{j}-\mathbf{K}_{21}^{j}\left(\mathbf{K}_{11}^{j}\right)^{-1} \mathbf{K}_{12}^{j}
\end{array}\right] .
\end{aligned}
$$

For simplicity, further analysis is conducted using the stiffness matrix formulation, although it is useful to note that the hybrid matrix formulation can be attractive for an analysis of the Floquet waves in periodic media due to the reported QZ eigenproblem factorization of the hybrid matrix (Tan, 2010). The whole layered or multilayered structure recursive stiffness matrix is (Rokhlin and Wang, 2002)

$$
\mathbf{K}^{J}=\left[\begin{array}{cc}
\mathbf{K}_{11}^{J-1}+\mathbf{K}_{12}^{J-1}\left(\mathbf{K}_{11}^{j}-\mathbf{K}_{22}^{J-1}\right)^{-1} \mathbf{K}_{21}^{J-1} & -\mathbf{K}_{12}^{J-1}\left(\mathbf{K}_{11}^{j}-\mathbf{K}_{22}^{J-1}\right)^{-1} \mathbf{K}_{12}^{j} \\
\mathbf{K}_{21}^{j}\left(\mathbf{K}_{11}^{j}-\mathbf{K}_{22}^{J-1}\right)^{-1} \mathbf{K}_{21}^{J-1} & \mathbf{K}_{22}^{j}-\mathbf{K}_{21}^{j}\left(\mathbf{K}_{11}^{j}-\mathbf{K}_{22}^{J-1}\right)^{-1} \mathbf{K}_{12}^{j}
\end{array}\right],
$$

where $\mathbf{K}^{J}$ is the whole stiffness matrix for the top $J$ layers, $\mathbf{K}^{J-1}$ is the total stiffness matrix for the $j-1$ layers. Using the whole stiffness matrix, the Lamb wave dispersion equation is given by

$$
\operatorname{det}\left(\mathbf{K}^{J}\right)=0 .
$$

Amplitude reflection and transmission coefficients for submersed or embedded elastic structures are given by (Rokhlin and Wang, 2002)

$$
\left[\begin{array}{c}
\mathbf{R}_{\mathrm{a}} \\
\mathbf{T}_{\mathrm{a}}
\end{array}\right]=\left[\begin{array}{c}
\mathbf{K}^{11} \mathbf{P}_{0}^{-}-\mathbf{D}_{0}^{-} \\
\mathbf{K}^{21} \mathbf{P}_{0}^{-}
\end{array}\right]\left[\begin{array}{cc}
-\mathbf{K}^{11} \mathbf{P}_{0}^{+}+\mathbf{D}_{0}^{+} & -\mathbf{K}^{12} \mathbf{P}_{N+1}^{-} \\
-\mathbf{K}^{21} \mathbf{P}_{0}^{+} & -\mathbf{K}^{22} \mathbf{P}_{N+1}^{-}+\mathbf{D}_{N+1}^{-}
\end{array}\right]^{-1},
$$


where $\mathbf{P}_{0, N+1}^{ \pm}, \mathbf{D}_{0, N+1}^{ \pm}$are the top and bottom semi-space terms. Explicit terms $\mathbf{P}_{0, N+1}^{ \pm}, \mathbf{D}_{0, N+1}^{ \pm}$for fluid semi-spaces are given in Rokhlin and Wang (2002). For a structure submersed in a fluid, the explicit amplitude reflection and transmission coefficients are given by

$$
\begin{aligned}
& \mathrm{R}_{\mathrm{a}}=\frac{\left(S_{11}^{33}-\Lambda\right)\left(S_{22}^{33}-\Lambda\right)-S_{21}^{33} S_{12}^{33}}{\left(S_{11}^{33}+\Lambda\right)\left(S_{22}^{33}-\Lambda\right)-S_{21}^{33} S_{12}^{33}}, \\
& \mathrm{~T}_{\mathrm{a}}=\frac{2 \Lambda S_{21}^{33}}{\left(S_{11}^{33}+\Lambda\right)\left(S_{22}^{33}-\Lambda\right)-S_{21}^{33} S_{12}^{33}},
\end{aligned}
$$

where $S_{i j}^{33}$ are the (3,3) elements in the matrix $\mathbf{S}_{i j}, \mathbf{S}=\mathbf{K}^{-1}$ is the compliance matrix (Rokhlin and Wang, 2002), $\Lambda=\cos \theta$ / $\left(i \omega \rho_{f} c_{f}\right)$, where $\rho_{\mathrm{f}}$ is the mass density of the fluid and $c_{\mathrm{f}}$ is the ultrasonic wave speed in the fluid. Energy reflection and transmission coefficients are calculated from Eqs. (18) and (19) taking square of the expressions.

\section{GUIDED WAVES IN A STRESSED TRI-LAYER}

A stressed layered structure, with a co-ordinate system for waves propagating in the $x-z$ plane is depicted in Fig. 2, comprising a polyvinylchloride (PVC) layer in between aluminum layers. Each layer is $0.1 \mathrm{~mm}$ thick. Material properties are listed in Table I (Gandhi et al., 2012; Korneev and Demčenko, 2014).

In our analysis, a static stress $\sigma_{22}$ was assumed to be constant and equal to $200 \mathrm{MPa}$ (tensile case), but varied in direction. In addition to showing the co-ordinate system for waves propagating in the $x-z$ plane, Fig. 2 also shows coordinate system for the stress using an unprimed coordinate system $(x$, $y, z)$, where the static stress is rotated through the angle $\phi$ via a rotational transformation (Gandhi et al., 2012).

Lamb wave phase velocity dispersion curves including shear horizontal modes in the stressed tri-layer are shown in Fig. 3. It is useful to note that the shear horizontal wave modes decouple from the sagittal wave motion when $\phi=0^{\circ}$ or $\phi=90^{\circ}$ or $\sigma_{22}=0 \mathrm{MPa}$. The solid dispersion curves are calculated when the static stress $\sigma_{22}=200 \mathrm{MPa}$ and the angle $\phi$ is $0^{\circ}, 45^{\circ}, 90^{\circ}$. The dashed curves show the guided wave dispersion curves in the unstressed tri-layer. In order to gain a better representation of the guided wave dispersion behaviour

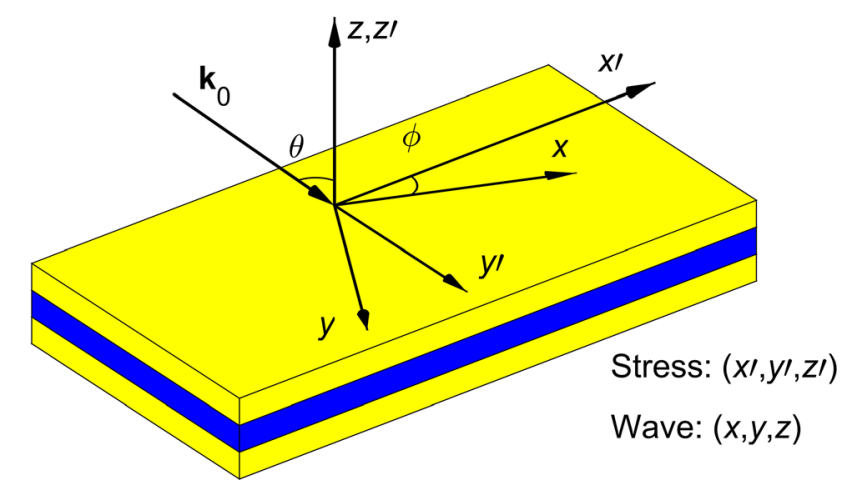

FIG. 2. (Color online) Stressed layered structure and co-ordinate system for waves propagating in the $x-z$ plane. $\mathbf{k}_{0}$ is the incident plane wave, $\theta$ is the incidence angle of the incident wave.
TABLE I. Material properties of elastic layers. The third order elastic constants are listed in Murnaghan's notation.

\begin{tabular}{lcccccc}
\hline \hline Material & $\lambda, \mathrm{GPa}$ & $\mu, \mathrm{GPa}$ & $l, \mathrm{GPa}$ & $m, \mathrm{GPa}$ & $n, \mathrm{GPa}$ & $\rho, \mathrm{kg} / \mathrm{m}^{3}$ \\
\hline Aluminum & 54.31 & 27.17 & -281.5 & -339 & -416 & 2704 \\
PVC & 3.88 & 1.63 & -33.43 & -20.88 & -15.86 & 1350 \\
\hline \hline
\end{tabular}

in the presence of the static stress, the dispersion curves are shown in separate figures, Figs. 3(b)-3(d), regarding to the wave mode type (symmetric, antisymmetric, and shear horizontal).

The results clearly indicate that the fundamental Lamb wave modes $\left(\mathrm{a}_{0}\right.$ and $\left.\mathrm{s}_{0}\right)$ show a low sensitivity both to the static stress and its direction up to $3 \mathrm{MHz}$. However, when the coupling occurs between the sagittal wave motion and shear horizontal modes, a significant change, not seen in the previously reported work (Gandhi et al., 2012) is observed in the $\mathrm{s}_{0}$ and $\mathrm{sh}_{0}$ phase velocities [see Figs. 3(b) and 3(d) when $\phi=45^{\circ}, 3-6 \mathrm{MHz}$ frequency range].

The fundamental shear horizontal wave mode $\mathrm{sh}_{0}$ shows a low sensitivity both to the static stress and direction in the low dispersion zone (below $3 \mathrm{MHz}$ ), although it does become sensitive to these in the higher dispersion zones (above $3 \mathrm{MHz}$ ). The results show that the higher order Lamb wave modes, at the same frequency, are more sensitive to the static stress and its direction than the fundamental modes. It is also apparent in Fig. 3(b) that the symmetric $\mathrm{s}_{1}$ mode is sensitive to both stress and direction, and that it has a higher sensitivity than the antisymmetric mode $a_{1}$ [Fig. 3(c)].

The shear horizontal $\mathrm{ah}_{1}$ mode, Fig. 3(d), also shows a sensitivity to both the static stress and its direction, close to the antisymmetric Lamb wave mode $\mathrm{a}_{1}$. However, the higher order modes, which are not included in our analysis, might also exist with high sensitivities to both static stress and its direction, although excitation and reception of these modes can be more complicated. The sensitivity to stress is not only the issue, as the wave mode should also be insensitive (or have low sensitivity) to possible variations in the material properties and the structure thickness. In future studies, we will present such a parametric study of the material properties.

\section{REFLECTION AND TRANSMISSION COEFFICIENTS FROM THE SUBMERSED STATICALLY STRESSED TRI-LAYER}

Ultrasonic contactless measurements possess a number of advantages when compared with contact measurements and is one reason why immersion and air-coupled ultrasonic measurements are used widely in scientific and industrial applications. As such both immersion and air-coupled ultrasonic techniques are attractive for evaluation of statically stressed structures.

Energy reflection $R$ and transmission $T$ coefficients from the submersed tri-layer structures in a fluid $c_{\mathrm{f}}=1480 \mathrm{~m} / \mathrm{s}$, $\rho_{\mathrm{f}}=1000 \mathrm{~kg} / \mathrm{m}^{3}$ are presented in Fig. 4 when the incident angle $\theta$ of the wave are $20^{\circ}, 40^{\circ}$ and $50^{\circ}$. The calculations are performed using a frequency step of $1 \mathrm{kHz}$, with the stress direction varying from $0^{\circ}$ to $90^{\circ}$ with a $5^{\circ}$ step. 

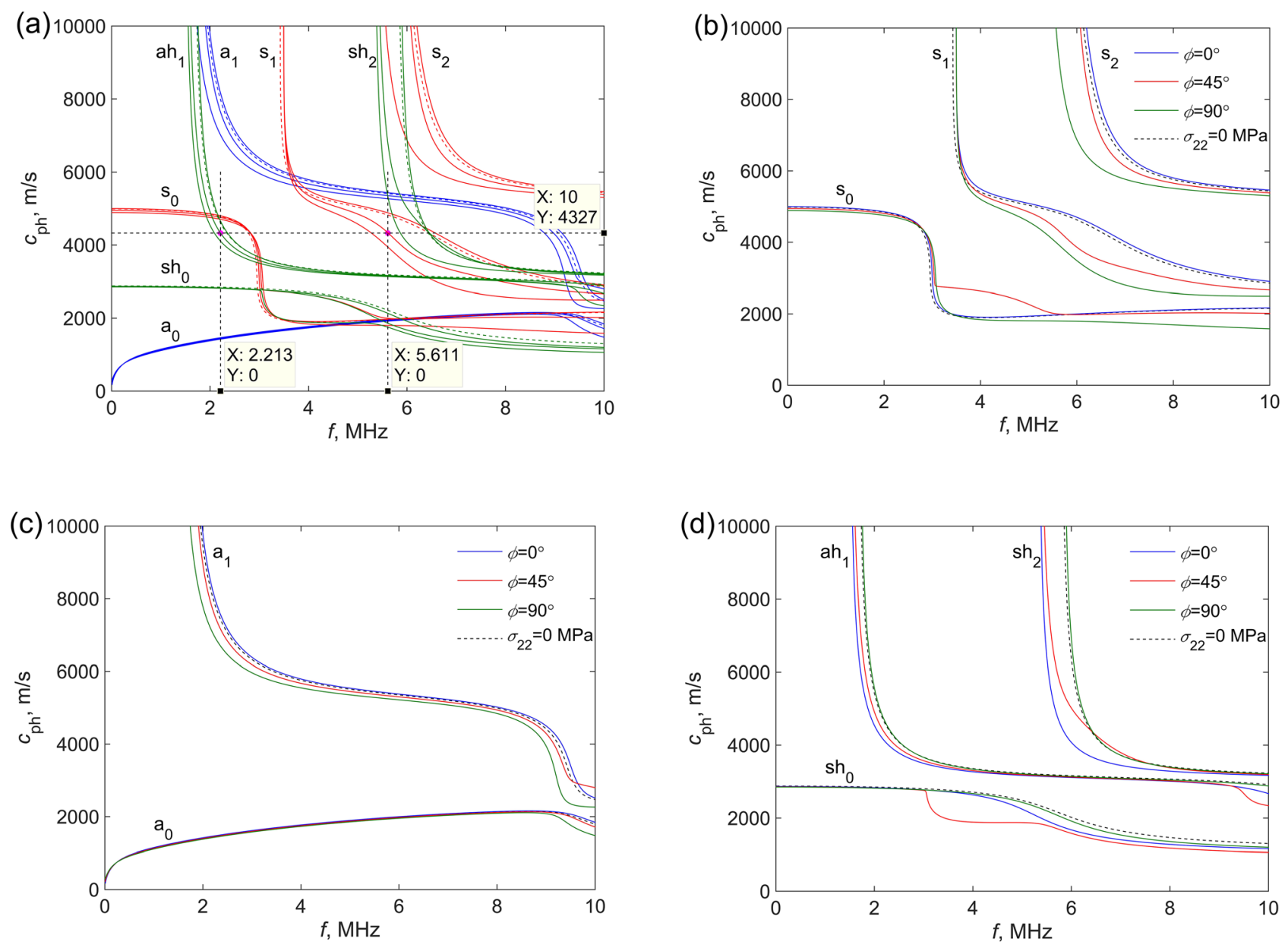

FIG. 3. (Color online) Guided wave dispersion curves in the stressed tri-layer when the static stress $\sigma_{22}=200 \mathrm{MPa}$ and the angle between the static stress and wave propagation direction $\phi$ is $0^{\circ}, 45^{\circ}, 90^{\circ}$ : (a) all modes, (b) symmetric modes, (c) antisymmetric modes, and (d) shear horizontal modes where sh and $a h$ represent the symmetric and antisymmetric shear horizontal wave modes, respectively. The dashed curves indicate the dispersion curves in the unstressed trilayer.
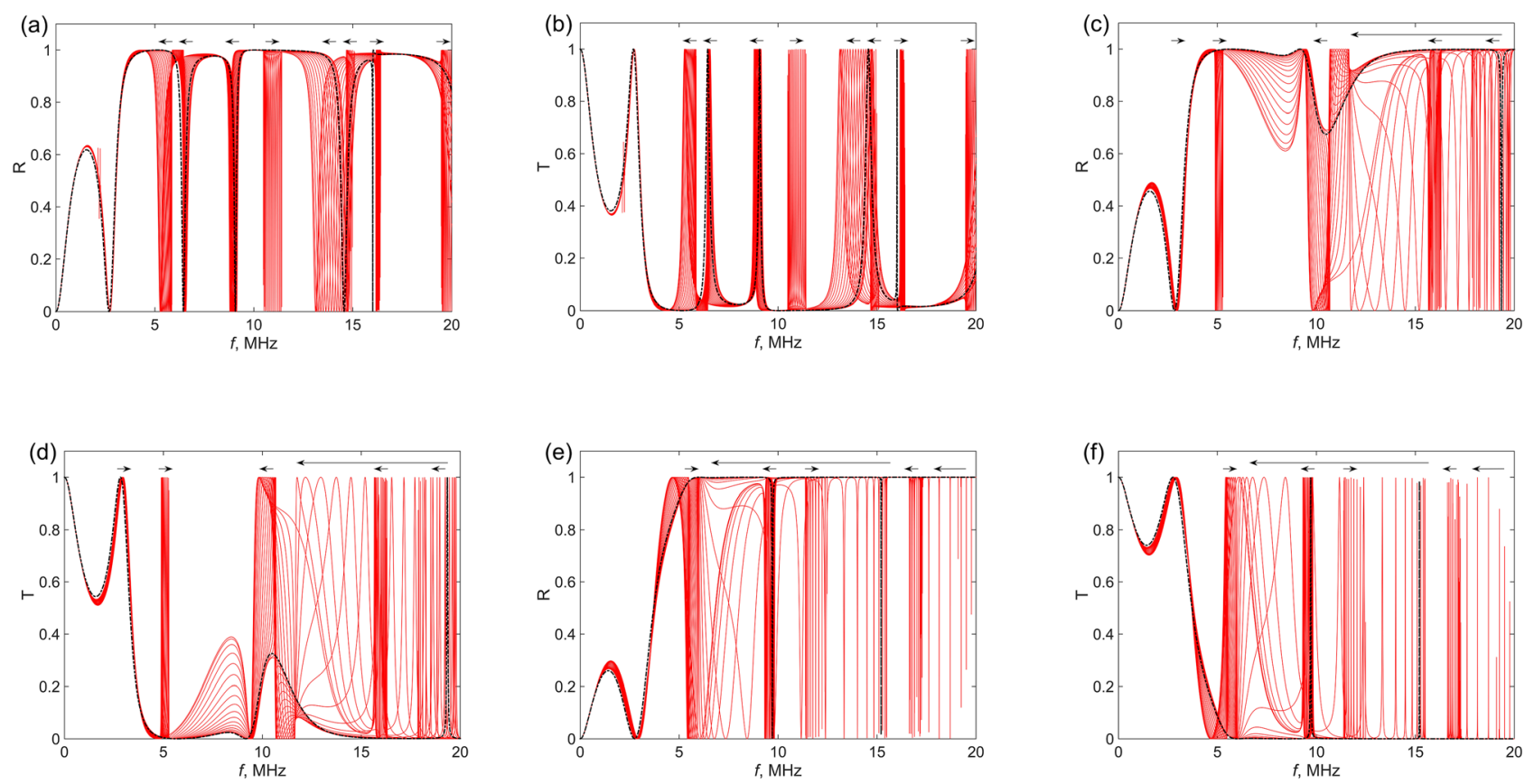

FIG. 4. (Color online) Energy reflection $R$ and transmission $T$ coefficients from the stressed tri-layer submersed in the fluid when $\sigma_{22}=200 \mathrm{MPa}$, the stress direction $\phi$ varies from $0^{\circ}$ to $90^{\circ}$ with a $5^{\circ}$ step and the wave incidence angle: (a) and (b) $\theta=20^{\circ}$, (c) and (d) $\theta=40^{\circ}$, and (e) and (f) $\theta=50^{\circ}$. Black dashed curves indicate the response from the unstressed tri-layer. Arrows indicate the peak movement direction regarding to the angle $\phi$. 
Figures 4(a) and 4(b) show significant changes in the ultrasonic response from this submersed statically stressed trilayer: reflection and transmission peaks are observed in the frequency ranges where they are not present in the unstressed layered structure, e.g., see frequencies 5.5 MHz and $11 \mathrm{MHz}$ in Figs. 4(a) and 4(b). Moreover, a shift of the peaks is observed due to the static stress direction change. When the incidence angle of the incident wave increases, $\theta=40^{\circ}$ or $\theta=50^{\circ}$, see Figs. 4(c)-4(f), the ultrasonic response from the submersed trilayer changes significantly from the case shown in Figs. 4(a) and 4(b). The results in Figs. 4(c) and 4(d) show that the reflection and transmission coefficient peaks lose their regularity. They are not concentrated around a certain frequency at higher frequencies $\left(\theta=40^{\circ}\right.$; above $\left.10 \mathrm{MHz}\right)$ which is observed in Figs. 4(a) and 4(b), hence an interpretation of data becomes more complicated. Significant and regular changes of the reflection and transmission coefficients are presented across a relatively wide frequency range $(5-10 \mathrm{MHz})$, as is attractive for practical measurements.

Further increase of the incident angle $\theta$ (from $40^{\circ}$ to $50^{\circ}$ ) makes the reflection and transmission coefficients more scattered [Figs. 4(e) and 4(f)]. These results in Fig. 4 clearly also show that there is an optimal wave incident angle for evaluation of the static or residual stresses in submersed layered structures.

Subsequently, we show a case study when the wave incidence angle is $\theta=20^{\circ}$, Fig. 5. Using Snell's law, one can see that ultrasonic wave velocity is $4327 \mathrm{~m} / \mathrm{s}$ in the aluminum layer of the tri= layer [see Fig. 3(a), horizontal dashed line and intersections with $\mathrm{ah}_{1}$ and $\mathrm{s}_{1}$ modes when $\phi=45^{\circ}$ ]. This velocity matches the velocities of the $\mathrm{ah}_{1}$ and $\mathrm{s}_{1}$ modes at $f=2.213 \mathrm{MHz}$ and $f=5.614 \mathrm{MHz}$, respectively.

As a consequence of the critical angle of incidence for these two modes, the corresponding peaks are observed in the reflection and transmission coefficients. Figure 5 shows peaks of both modes. It is seen that the peak corresponding to the $\mathrm{ah}_{1}$ is low due to the weak coupling of the shear horizontal wave motion to the sagittal wave motion. Figure 4(b) shows that at certain stress directions the coupling between the shear

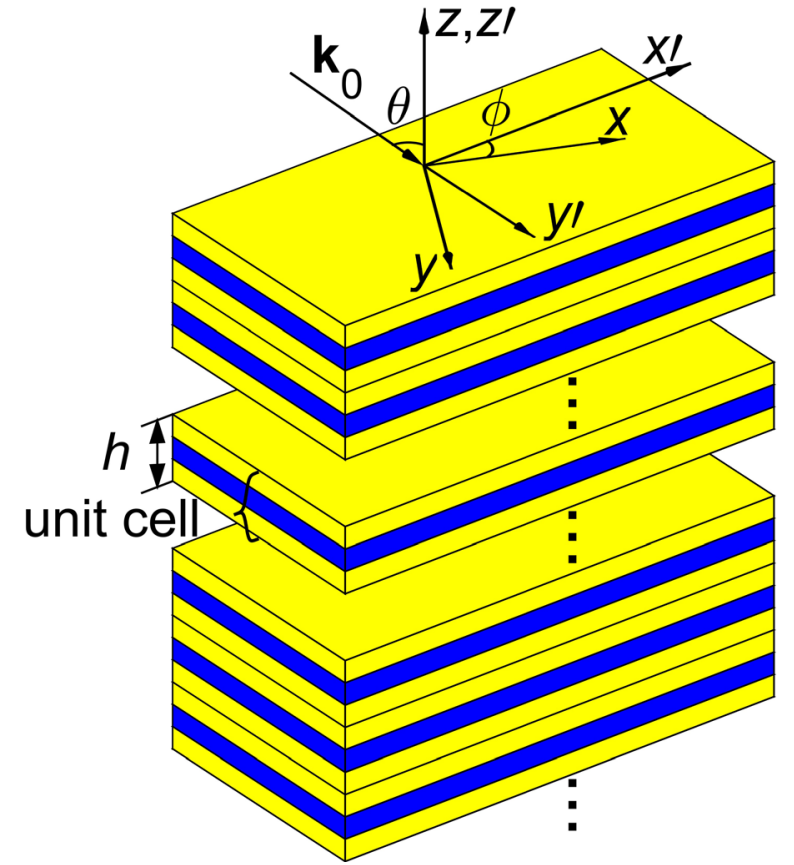

FIG. 6. (Color online) Periodic structure and co-ordinate system for waves propagating in the $x-z$ plane. $\mathbf{k}_{0}$ is the incident plane wave, $\theta$ is the incidence angle of the incident wave, $h$ is the thickness of the periodic unit cell.

horizontal and sagittal wave motions increases-higher peaks are present in the ultrasonic response. The results, as seen in Fig. 5(a), also show a high efficiency transmission of the ultrasonic wave (reflection coefficient is almost 0 ) when the incident angle $\theta$ matches the critical angle of the $\mathrm{s}_{1}$ mode.

\section{FLOQUET WAVES IN A PERIODIC STRESSED MEDIA}

When a multilayered medium contains a periodicity (various composite and 1D phononic structures, Fig. 6), Floquet wave theory can be used for elastic wave analysis.

This theory is useful for homogenization of multilayered media, and for the analysis of 1D phononic structures in terms of their band-gaps (Demčenko et al., 2018). When a (a)

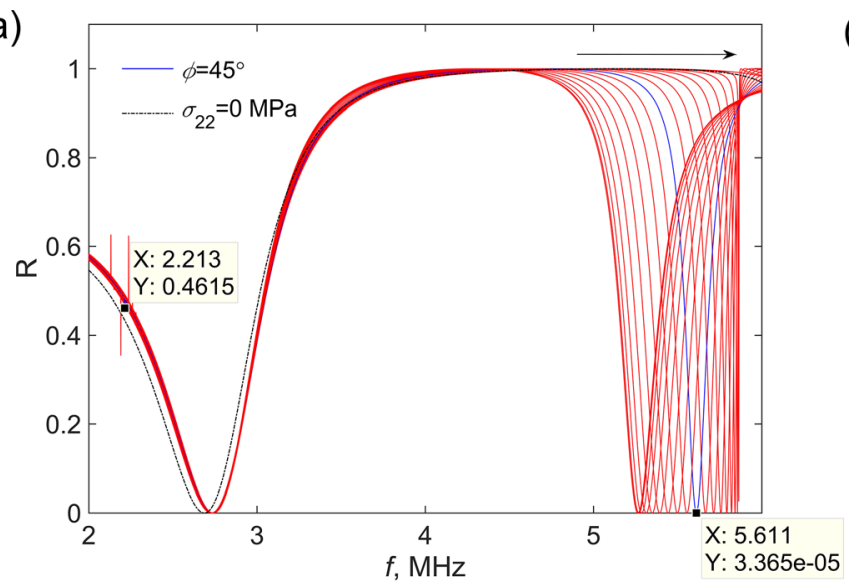

(b)

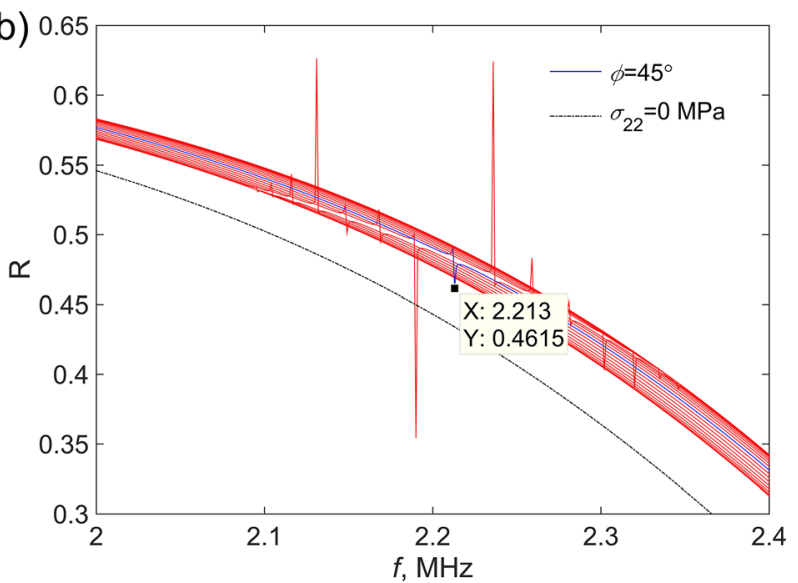

FIG. 5. (Color online) Energy reflection coefficient $R$ when the wave incidence angle $\theta=20^{\circ} ; \sigma_{22}=200 \mathrm{MPa}$, when the stress direction $\phi$ varies from $0^{\circ}$ to $90^{\circ}$ with a $5^{\circ}$ step. Blue curves indicate the response from the submersed tri-layer when $\phi=45^{\circ}$. Two typical points at frequencies $2.213 \mathrm{MHz}$ (ah ${ }_{1}$ mode) and $5.614 \mathrm{MHz}\left(\mathrm{s}_{1}\right.$ mode) are shown in (a). Arrow indicates the peak movement direction regarding to the angle $\phi$. Illustration of coupling of the shear horizontal wave mode to sagittal wave motion (b). 
periodic structure is statically stressed, effective second and third order elastic constants can be estimated by applying the Floquet wave theory. For infinite periodic medium the periodicity requires (Wang and Rokhlin, 2002a)

$$
\left[\begin{array}{c}
\mathbf{u}^{+} \\
\boldsymbol{\sigma}^{+}
\end{array}\right]=e^{\mathrm{i} k_{\mathrm{zF}} h}\left[\begin{array}{l}
\mathbf{u}^{-} \\
\boldsymbol{\sigma}^{-}
\end{array}\right],
$$

where $k_{z \mathrm{~F}}$ is the vertical Floquet wavenumber, $h$ is the thickness of the periodic unit cell, $\mathbf{u}^{ \pm}, \boldsymbol{\sigma}^{ \pm}$are the displacements and stresses at cell top $(+)$ and bottom $(-)$ surfaces, respectively. Using the recursive stiffness matrix approach, the Floquet wave equation for the infinite periodic medium is written in this form (Wang and Rokhlin, 2002a)

$$
\left(e^{\mathrm{i} k_{\mathrm{z}} h} \mathbf{K}_{\mathrm{c}}^{21}-e^{-i k_{z \mathrm{~F}} h} \mathbf{K}_{\mathrm{c}}^{12}+\mathbf{K}_{\mathrm{c}}^{22}-\mathbf{K}_{\mathrm{c}}^{11}\right) \mathbf{u}^{-}=0,
$$

where $\mathbf{K}_{c}$ is the whole stiffness matrix of the unit periodic cell. $\mathbf{u}^{-}$is the Floquet wave unit displacement vector and is equivalent to the displacement polarization $\mathbf{p}$, see Eq. (1). Equation (21) is equivalent to the Christoffel equation, see
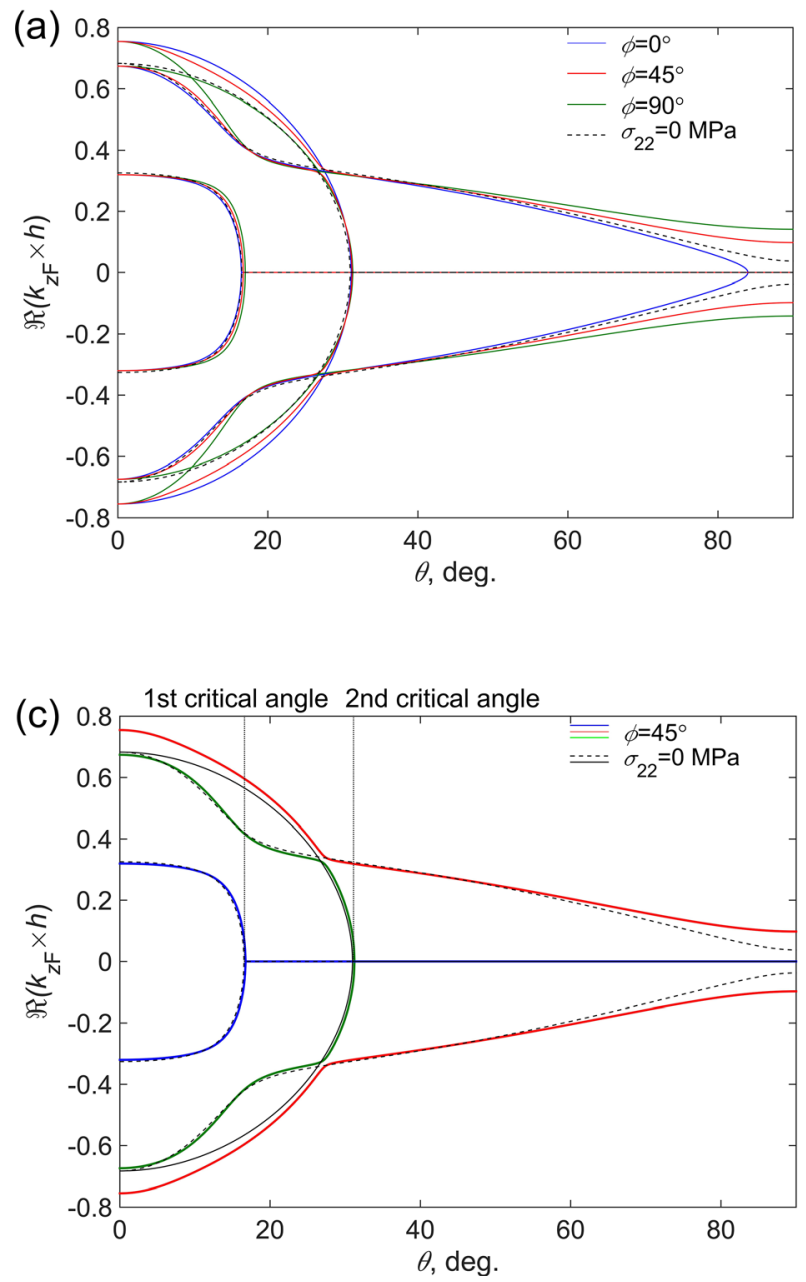

Eq. (2), and it is solved for the Floquet wavenumbers (eigenvalues), setting the determinant to 0 ,

$$
A_{3} \cos \left(3 k_{z \mathrm{~F}} h\right)+A_{2} \cos \left(2 k_{z \mathrm{~F}} h\right)+A_{1} \cos \left(k_{z \mathrm{~F}} h\right)+A_{0}=0,
$$

where $A_{i}$ are the coefficients formed by the $\mathbf{K}_{c}$ elements. We confirm that some of the reported coefficients (Wang and Rokhlin, 2002a) are incorrect (Ishii and Biwa, 2015). The updated coefficients are given by

$$
\begin{aligned}
A_{3}= & \operatorname{det}\left(\mathbf{K}_{\mathrm{c}}^{21}\right) \\
A_{2}= & 1 / 2\left(\operatorname{det}\left(\mathbf{M}+\mathbf{K}_{\mathrm{c}}^{21}\right)+\operatorname{det}\left(\mathbf{M}-\mathbf{K}_{\mathrm{c}}^{21}\right)\right)-\operatorname{det}(\mathbf{M}) \\
A_{1}= & 1 / 2\left(\operatorname{det}\left(\mathbf{M}+\mathbf{K}_{\mathrm{c}}^{21}\right)-\operatorname{det}\left(\mathbf{M}+\mathbf{K}_{\mathrm{c}}^{12}\right)\right. \\
& \left.+\operatorname{det}\left(\mathbf{K}_{\mathrm{c}}^{21}-\mathbf{K}_{\mathrm{c}}^{12}\right)\right)-2 \operatorname{det}\left(\mathbf{K}_{\mathrm{c}}^{21}\right) \\
A_{0}= & 1 / 4\left(\operatorname{det}\left(\mathbf{M}+\mathbf{K}_{\mathrm{c}}^{12}-\mathbf{K}_{\mathrm{c}}^{21}\right)\right. \\
& \left.+\operatorname{det}\left(\mathbf{M}-\mathbf{K}_{\mathrm{c}}^{12}+\mathbf{K}_{\mathrm{c}}^{21}\right)\right)-A_{2}
\end{aligned}
$$
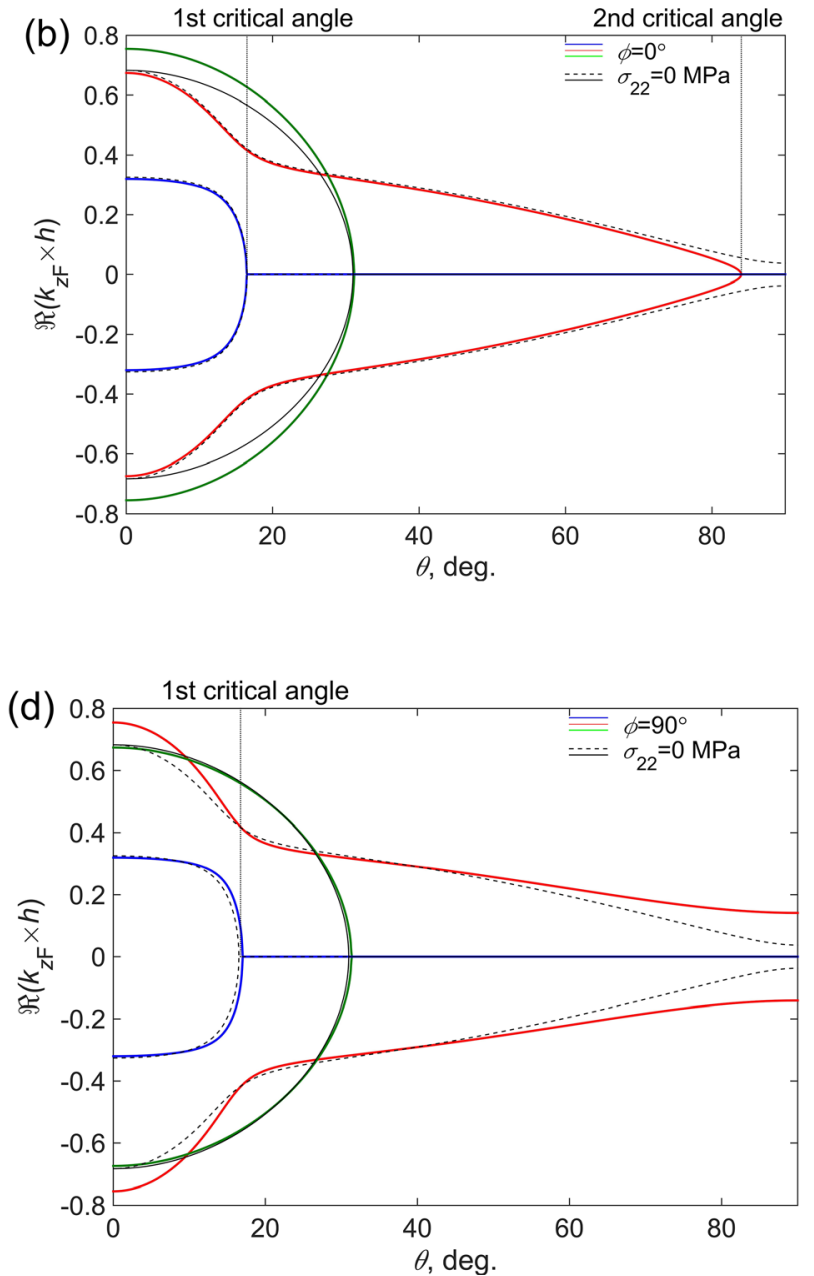

FIG. 7. (Color online) Effective homogeneous periodic semi-space response when $\sigma_{22}=200 \mathrm{MPa}$ and $f=0.5 \mathrm{MHz}$. Real part of the Floquet wavenumber and unit cell thickness product $k_{z \mathrm{~F}} \times h$ : (a) all wavenumbers, (b) wavenumbers when $\phi=0^{\circ}$, (c) wavenumbers when $\phi=45^{\circ}$, and (d) wavenumbers when $\phi=90^{\circ}$. Black curves indicate response from the unstressed semi-space. Dotted vertical lines show position of critical angles. Positions of critical angles for uncoupled modes are not highlighted. 
where $\mathbf{M}=\mathbf{K}_{\mathrm{c}}^{22}-\mathbf{K}_{\mathrm{c}}^{11}$. It is important to note that the updated coefficients are different by one sign from the reported in Ishii and Biwa (2015). Using these coefficients, reported results were repeated (Demčenko et al., 2018).

When the Floquet wavenumbers are known, the corresponding unit polarization vectors $\mathbf{p}_{j}^{\mathrm{F} \pm}$ (eigenvectors) are found from Eq. (21). The multi-mode region of the Floquet waves require a correct sorting of the eigenvalues and eigenvectors (Marini et al., 2010). Also, we found that a singular value decomposition is preferable for finding the non-trivial solutions of the homogeneous equation, see Eq. (21). Finally, the stress vector of the unit cell is found from the following expression (Wang and Rokhlin, 2002b):

$$
\mathbf{d}_{j}^{\mathrm{F} \pm}=\left(\mathbf{K}_{\mathrm{c}}^{11}+e^{ \pm \mathrm{i} k_{z} h} \mathbf{K}_{\mathrm{c}}^{12}\right) \mathbf{p}_{j}^{\mathrm{F} \pm}
$$

The amplitude reflection coefficient from a submersed semispace in terms of the Floquet wave equation parameters is written in the following form (Wang and Rokhlin, 2002b)

$$
\mathrm{R}_{\mathrm{as}}=\frac{S_{\mathrm{F}}^{33}-\Lambda}{S_{\mathrm{F}}^{33}+\Lambda},
$$

where $S_{\mathrm{F}}^{33}$ is the $(3,3)$ element in the $3 \times 3$ surface compliance matrix for a homogeneous or layered anisotropic semi-space.
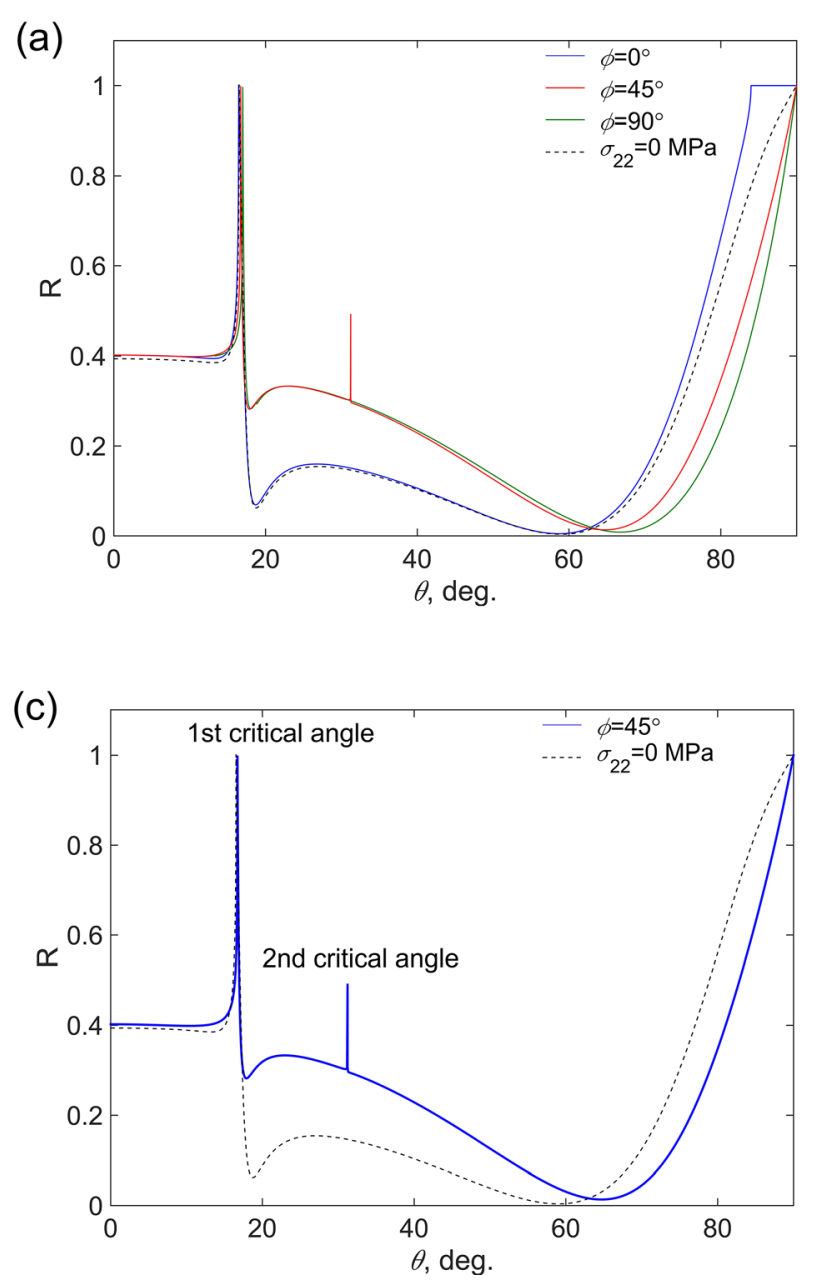

The compliance matrix can be calculated from the Floquet wave displacement and stress vectors and it is given by $\mathbf{S}_{\mathrm{F}}=\mathbf{P}_{\mathrm{F}}^{-}\left(\mathbf{D}_{\mathrm{F}}^{-}\right)^{-1}$.

\section{ULTRASONIC WAVE RESPONSE FROM A SUBMERSED PERIODIC SEMI-SPACE IN A PRESENCE OF THE STATIC STRESS}

Our Floquet wave analysis is performed for a unit periodic cell shown in Fig. 6 which considers three layers. The incidence wave angle $\theta$ varies from $0^{\circ}$ to $90^{\circ}$ with a step of $0.001^{\circ}$. The static stress is $\sigma_{22}=200 \mathrm{MPa}$, the angle $\phi$ is $0^{\circ}$, $45^{\circ}$, and $90^{\circ}$. Two cases are presented: ultrasonic response from effective homogeneous (Figs. 7 and 8) and inhomogeneous (Figs. 9 and 10) semi-spaces.

Figure 7 shows the real part of the product $k_{z \mathrm{~F}} \times h$ for the effective homogeneous semi-space response when the wave incidence frequency is $0.5 \mathrm{MHz}$. One can see that the Floquet wavenumbers are equal to the wavenumbers of plane elastic waves in an effective medium. It can also be seen that the Floquet vertical wavenumbers and partial wavenumbers in effective homogeneous media are identical (Wang and Rokhlin, 2002a). The corresponding ultrasonic
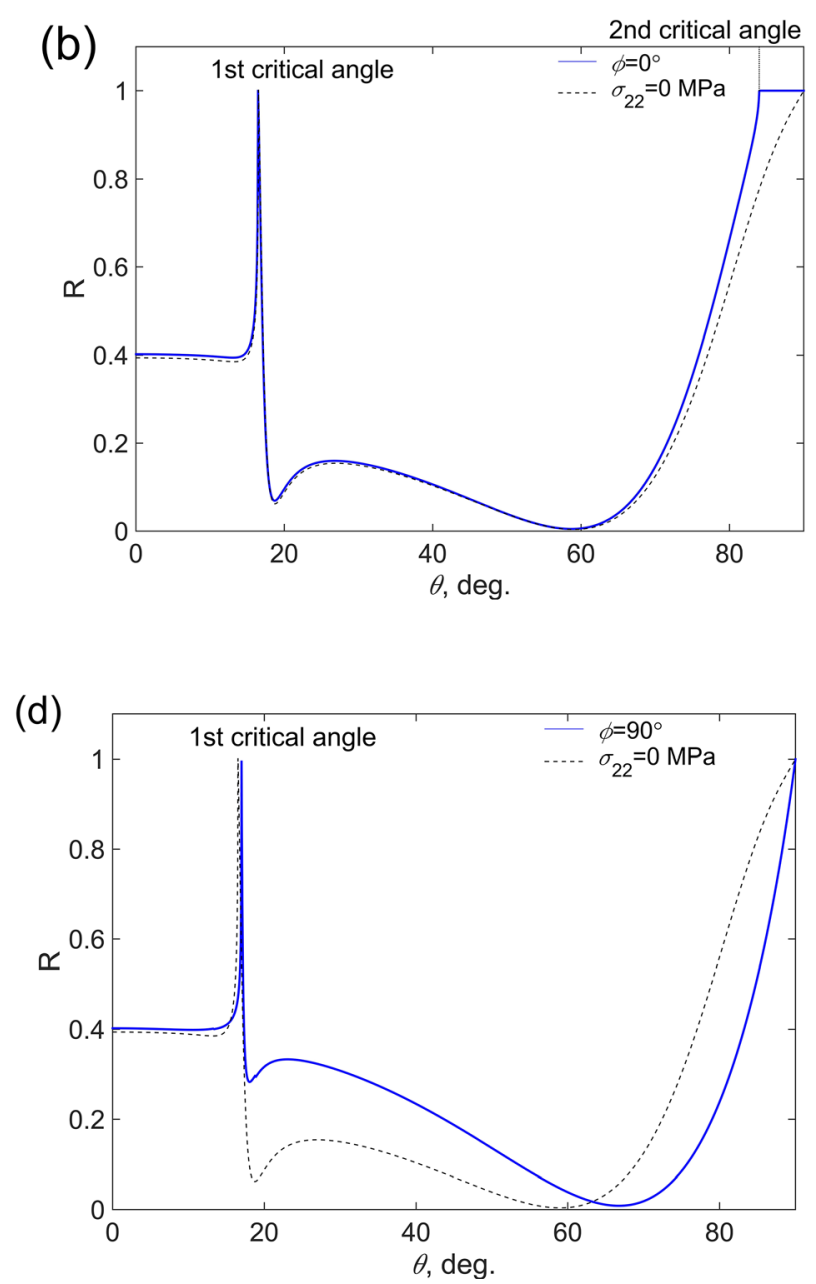

FIG. 8. (Color online) Ultrasonic response from the effective homogeneous periodic semi-space submersed in the fluid when $\sigma_{22}=200 \mathrm{MPa}$ and $f=0.5 \mathrm{MHz}$. Energy reflection coefficients from the periodic semi-space submersed in the fluid: (a) all reflection coefficients, $(\mathrm{b})$ reflection coefficient when $\phi=0^{\circ},(\mathrm{c})$ reflection coefficient when $\phi=45^{\circ}$, and (d) reflection coefficient when $\phi=90^{\circ}$. Black curves indicate response from the unstressed semi-space. 
(a)
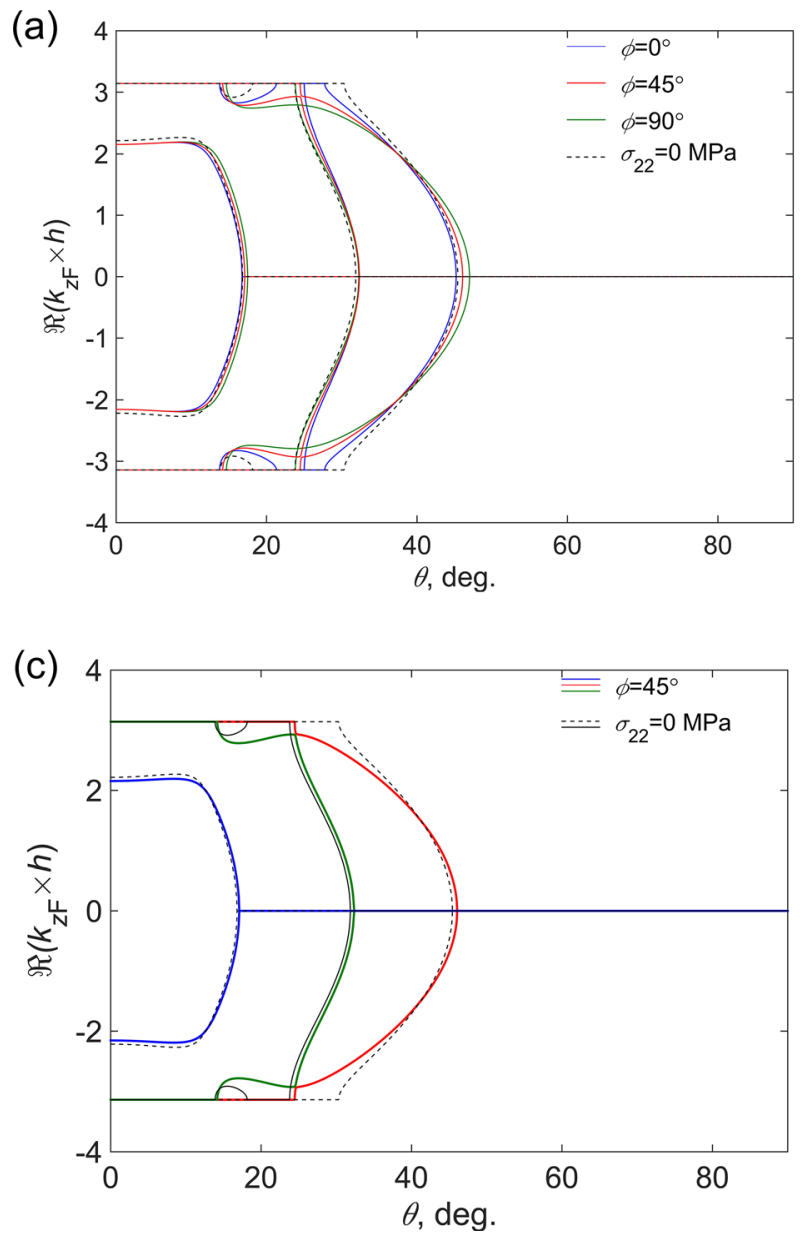

(b)

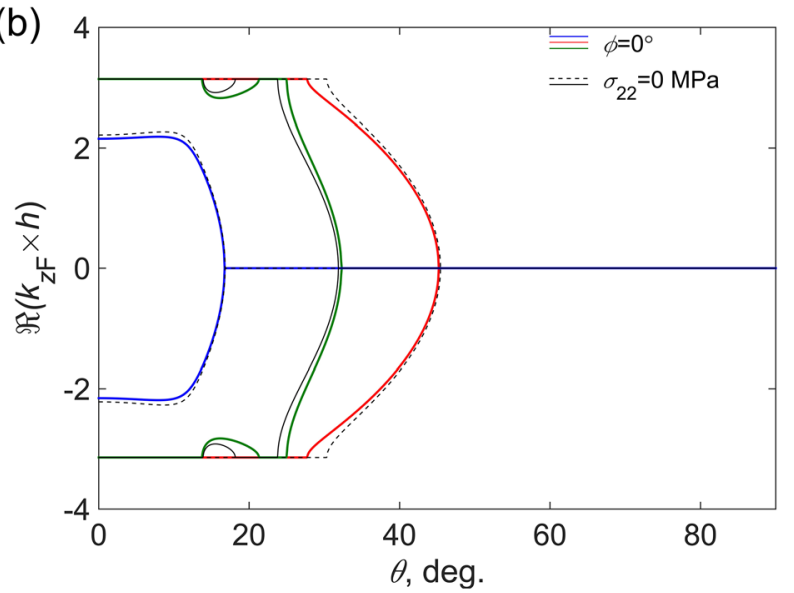

(d)

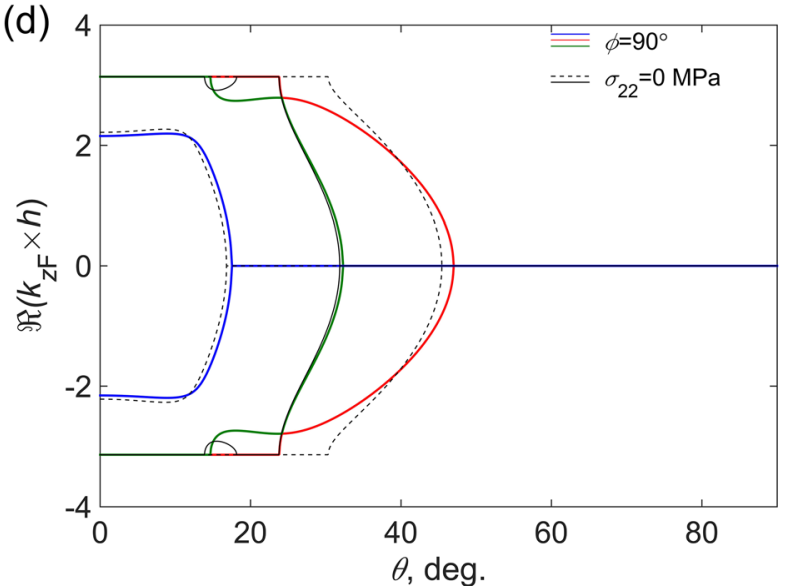

FIG. 9. (Color online) Inhomogeneous periodic semi-space response when $\sigma_{22}=200 \mathrm{MPa}$ and $f=3 \mathrm{MHz}$. Real part of the Floquet wavenumber and unit cell thickness product $k_{z \mathrm{~F}} \times h$ : (a) all wavenumbers, (b) wavenumbers when $\phi=0^{\circ}$, (c) wavenumbers when $\phi=45^{\circ}$, and (d) wavenumbers when $\phi=90^{\circ}$. Black curves indicate response from the unstressed semi-space.

wave energy reflection coefficient $\mathrm{R}$ from the submersed periodic semi-space is shown in Fig. 8.

Decomposed results regarding to the angle $\phi$ are depicted in Figs. 7(b) and 7(c) and 8(b) and 8(c). When the angle $\phi=0^{\circ}$, the results show [see Fig. 7(b)] that the Floquet wavenumbers which are equal to quasi-longitudinal partial waves (blue colour) have a very low sensitivity to the static stress. This is readily seen in the energy reflection coefficient, Fig. 8 (b), below the first critical angle $\left(\theta=16.5^{\circ}\right)$. The reflection coefficient above the first critical angle also shows a low sensitivity to the static stress, although, a response at a second critical angle occurs (approximately $84^{\circ}$; this angle is not present in the unstressed structure). This confirms that the Floquet wavenumbers, which are equal to quasi-shear partial waves (vertical polarization; red curves), also exhibit a low sensitivity to the static stress. The Floquet wavenumbers, which are equal to pure shear horizontal partial waves (green curves), are not observed in the reflection coefficient due to their decoupling from the sagittal wave motion.

When the angle $\phi=45^{\circ}$ [Figs. 7(c) and 8(c)], the results are different from the case analysed above in Figs. 7(b) and 8(b). A coupling of the Floquet wavenumbers, which are equal to the shear horizontal partial waves (green curves) to the sagittal wave motion, is observed in the response [Fig. 7(c)]. The second critical angle of the fast quasi-shear wave is observed at the angle $\theta=31.2^{\circ}$. A larger value change in the energy reflection coefficient is observed above the first critical angle $\left(\theta=16.7^{\circ}\right)$ than in the case when $\phi=0^{\circ}$.

Figures 7(d) and 8(d) depicts the case when $\phi=90^{\circ}$. The Floquet wavenumbers, which are equal to pure shear horizontal partial waves [green curves, Fig. 7(d)], are decoupled from the sagittal wave motion, hence they do not affect the energy reflection coefficient [Fig. 8(d)]. In the analysed case, only the first critical angle is reached at $\left(\theta=17^{\circ}\right)$, which corresponds to the Floquet wavenumbers (blue curves) equivalent to quasi-longitudinal partial waves.

The results in Figs. 7(d) and 8(d) show that the most significant shift of the first critical angle is observed when $\phi=90^{\circ}$. However, this shift is small and it is $0.5^{\circ}$. The energy reflection coefficient above the first critical angle, Fig. 8(d), shows behaviour close to the case when $\phi=45^{\circ}$, except that the second critical angle is not observed in the energy reflection curve, see Fig. 8(c).

An increase of the incident wave frequency results in a loss of homogenisation domain. A procedure for estimation of the homogenisation domain is reported in Wang and Rokhlin (2002a). Figures 9 and 10 show these results for the real part of the product $k_{z \mathrm{~F}} \times h$ and energy reflection coefficients, respectively, when the incident wave frequency is 
(a)

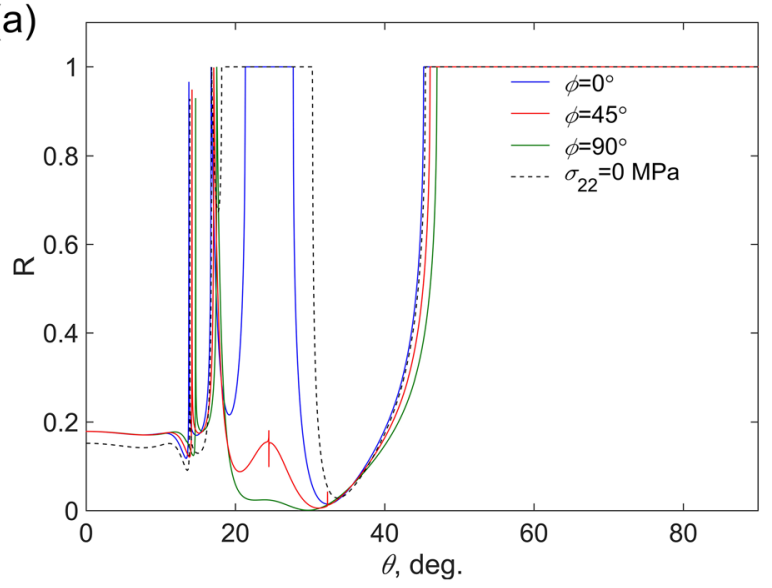

(c)

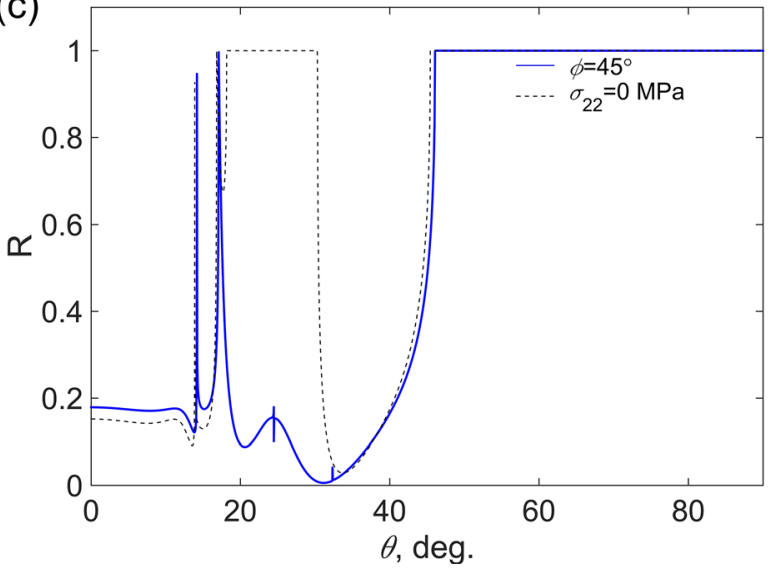

(b)

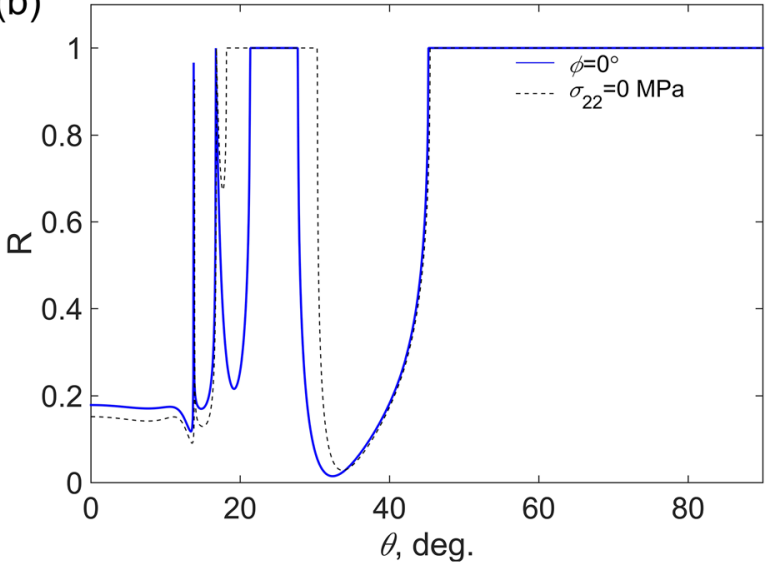

(d)

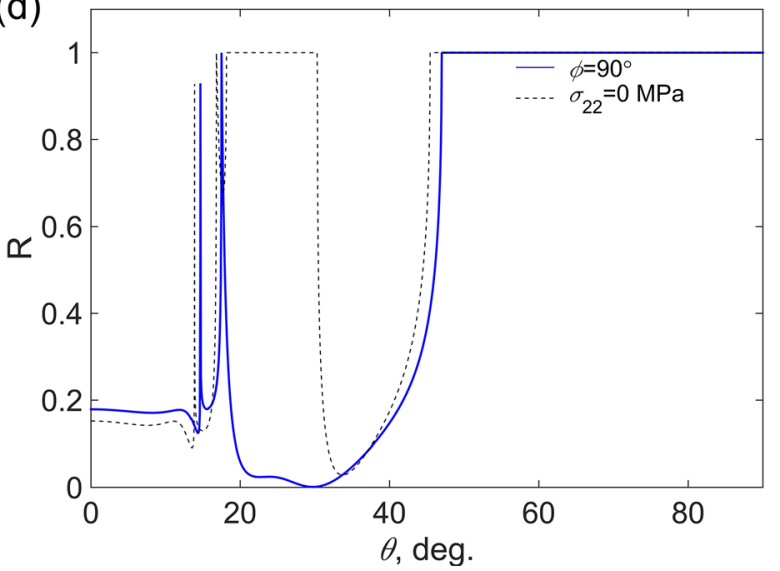

FIG. 10. (Color online) Ultrasonic response from the inhomogeneous periodic semi-space submersed in the fluid when $\sigma_{22}=200 \mathrm{MPa}$ and $f=0.5 \mathrm{MHz}$. Energy reflection coefficients from the periodic semi-space submersed in the fluid: (a) all reflection coefficients, (b) reflection coefficient when $\phi=0^{\circ}$, (c) reflection coefficient when $\phi=45^{\circ}$, and (d) reflection coefficient when $\phi=90^{\circ}$. Black curves indicate response from the unstressed semi-space.

$3 \mathrm{MHz}$. In this case, ultrasonic response is observed from the inhomogeneous periodic semi-space. The results show a complex response and a limited range (approximately up to $47^{\circ}$ ) of possible wave incidence angles that the incident wave would penetrate the semi-space. Above this angle, $47^{\circ}$, all energy is reflected back.

The reflection coefficient shows that there is a band-gap when the semi-space is unstressed (see the wave incidence range $18^{\circ}-30^{\circ}$ in Fig. 10). When the angle $\phi=0^{\circ}$, the bandgap width reduces [Fig. 10(b)], and it disappears when $\phi=45^{\circ}$ and $\phi=90^{\circ}$ [Figs. 10(c) and 10(d)]. The results in Figs. 10(c) and 10(d) show that there is a small difference between the reflection coefficients, a more significant difference is observed in the incidence angle range $20^{\circ}-30^{\circ}$ (approximately band-gap range).

An analysis of short wavelength range is reported in Demčenko et al. (2018). It is shown that band-gaps are tunable by the application of a static stress which is dependent both on magnitude and direction.

\section{CONCLUSIONS}

Reformulation of the stiffness matrix method for analysis of the elastic wave velocities as a function of applied static stress in multilayered and 1D phononic structures has been presented in this work. The method was used for the analysis of guided wave phase velocity dispersion curves in a statically stressed tri-layered structure. The approach was also used to address the analysis of energy reflection and transmission coefficients from a submersed statically stressed tri-layer. The analysis showed that higher order guided wave modes at the same frequency are more sensitive to static stress and its direction than the fundamental modes.

Using Floquet wave theory, a statically stressed semiinfinite periodic media was also analyzed in terms of the Floquet wavenumbers and energy reflection coefficients from the fluid loaded statically stressed semi-space. The analysis showed that a small shift of the first critical angle was expected in the energy reflection coefficient from the statically stressed effective homogeneous periodic semispace. The most significant change of the energy reflection coefficient was observed in the absolute value just above the first critical angle. When homogenization domain was lost, the ultrasonic response became complex. Finally, the analysis showed that a band-gap strongly depends on the stress direction and this band-gap can disappear due to the static stress. 


\section{ACKNOWLEDGMENTS}

J.M.C. acknowledges EPSRC Fellowship (EP/K027611/1) and the ERC advanced investigator award (340117Biophononics). All data created during this research are openly available from the University of Glasgow at http://dx.doi.org/ 10.5525/gla.researchdata.626.

Braga, A., and Herrmann, G. (1992). "Floquet waves in anisotropic periodically layered composites," J. Acoust. Soc. Am. 91(3), 1211-1227.

Demčenko, A., Mazilu, M., Wilson, R., Volker, A. W. F., and Cooper, J. M. (2018). "Hyperelastic tuning of one dimensional phononic band-gaps using directional stress," IEEE Trans. Ultrason. Ferroelectr. Frequency Control 65(6), 1056-1061.

Demčenko, A., Žukauskas, E., Kažys, R., and Voleišis, A. (2006). "Interaction of the a0 lamb wave mode with a de-lamination type defect in glare 3-3/2 composite material," Acta Acust. united Acust. 92(4), 540-548.

Dubuc, B., Ebrahimkhanlou, A., and Salamone, S. (2017). "Effect of pressurization on helical guided wave energy velocity in fluid-filled pipes," Ultrasonics 75, 145-154.

Egle, D., and Bray, D. (1976). "Measurement of acoustoelastic and thirdorder elastic constants for rail steel," J. Acoust. Soc. Am. 60(3), 741-744.

Galich, P., Fang, N., Boyce, M., and Rudykh, S. (2017). "Elastic wave propagation in finitely deformed layered materials," J. Mech. Phys. Solids 98, 390-410.

Gandhi, N., Michaels, J., and Lee, S. (2012). "Acoustoelastic Lamb wave propagation in biaxially stressed plates," J. Acoust. Soc. Am. 132(3), $1284-1293$

Gennisson, J.-L., Rénier, M., Catheline, S., Barriére, C., Bercoff, J., Tanter, M., and Fink, M. (2007). "Acoustoelasticity in soft solids: Assessment of the nonlinear shear modulus with the acoustic radiation force," J. Acoust. Soc. Am. 122(6), 3211-3219.

Hughes, D., and Kelly, J. (1953). "Second-order elastic deformation of solids," Phys. Rev. 92(5), 1145-1149.

Ishii, Y., and Biwa, S. (2015). "Transmission of ultrasonic waves at oblique incidence to composite laminates with spring-type interlayer interfaces," J. Acoust. Soc. Am. 138(5), 2800-2810.

Kino, G., Barnett, D., Grayeli, N., Herrmann, G., Hunter, J., Ilić, D., Johnson, G., King, R., Scott, M., Shyne, J., and Steele, C. (1980). "Acoustic measurements of stress fields and microstructure," J. Nondestruct. Eval. 1(1), 67-77.

Korneev, V., and Demčenko, A. (2014). "Possible second-order nonlinear interactions of plane waves in an elastic solid," J. Acoust. Soc. Am. 135(2), 591-598.

Korneev, V., and Glubokovskikh, S. (2013). "Seismic velocity changes caused by an overburden stress," Geophysics 78(5), WC25-WC31.

Kubrusly, A., Braga, A., and Von, D. W. (2016). "Derivation of acoustoelastic Lamb wave dispersion curves in anisotropic plates at the initial and natural frames of reference," J. Acoust. Soc. Am. 140(4), 2412-2417.

Kushwaha, M., Halevi, P., Dobrzynski, L., and Djafari-Rouhani, B. (1993). "Acoustic band structure of periodic elastic composites," Phys. Rev. Lett. 71(13), 2022-2025.
Lee, J., and Soutis, C. (2007). "A study on the compressive strength of thick carbon fibre-epoxy laminates," Compos. Sci. Technol. 67(10), 2015-2026.

Li, Y., Zhou, X., Bian, Z., Xing, Y., and Song, J. (2017). "Thermal tuning of the interfacial adhesive layer on the band gaps in a one-dimensional phononic crystal," Compos. Struct. 172, 311-318.

Marini, S., Coves, A., Boria, V. E., and Gimeno, B. (2010). "Full-wave modal analysis of slow-wave periodic structures loaded with elliptical waveguides," IEEE Trans. Electron Dev. 57(2), 516-524.

Mignogna, R. B. (1989). "General analytic solution of the wave equation for anisotropic materials using symbolic manipulation," in Review of Progress in Quantitative Nondestructive Evaluation, edited by D. O. Thompson and D. E. Chimenti (Springer, New York), pp. 133-140.

Mseddi, S., Njeh, A., and Hédi, B. G. (2014). "Acoustoelastic effects in anisotropic layered structure of $\mathrm{Cu} / \mathrm{Si}(001)$," Mech. Adv. Mater. Struct. 21(9), 710-715.

Osetrov, A., Fröhlich, H.-J., Koch, R., and Chilla, E. (2000). "Acoustoelastic effect in anisotropic layered structures," Phys. Rev. B 62(21), 13963-13969.

Pao, Y.-H., and Gamer, U. (1985). "Acoustoelastic waves in orthotropic media,” J. Acoust. Soc. Am. 77(3), 806-812.

Pei, N., and Bond, L. (2016). "Higher order acoustoelastic Lamb wave propagation in stressed plates," J. Acoust. Soc. Am. 140(5), 3834-3843.

Potel, C., Gatignol, P., and De, B. (2001). "Energetic criterion for the radiation of Floquet waves in infinite anisotropic periodically multilayered media," Acta Acust. united Acust. 87(3), 340-351.

Qu, J. M., and Liu, G. L. (1998). Effects of Residual Stress on Guided Waves in Layered Media (Plenum Press, New York).

Rokhlin, S., Bolland, T., and Adler, L. (1986). "Reflection and refraction of elastic waves on a plane interface between two generally anisotropic media," J. Acoust. Soc. Am. 79(4), 906-918.

Rokhlin, S., and Wang, L. (2002). "Stable recursive algorithm for elastic wave propagation in layered anisotropic media: Stiffness matrix method," J. Acoust. Soc. Am. 112, 822-834.

Tan, E. (2006). "Hybrid compliance-stiffness matrix method for stable analysis of elastic wave propagation in multilayered anisotropic media," J. Acoust. Soc. Am. 119(1), 45-53.

Tan, E. (2010). "Generalized eigenproblem of hybrid matrix for Floquet wave propagation in one-dimensional phononic crystals with solids and fluids," Ultrasonics 50(1), 91-98.

Wang, L., and Rokhlin, S. (2002a). "Floquet wave homogenization of periodic anisotropic media," J. Acoust. Soc. Am. 112(1), 38-45.

Wang, L., and Rokhlin, S. (2002b). "Time-resolved line focus acoustic microscopy of layered anisotropic media: Application to composites," IEEE Trans. Ultrason. Ferroelectr. Frequency Control 49(9), 1231-1244.

Wang, P., Casadei, F., Shan, S., Weaver, J. C., and Bertoldi, K. (2014). "Harnessing buckling to design tunable locally resonant acoustic metamaterials," Phys. Rev. Lett. 113(1), 014301.

Zhang, H., Kosinski, J., and Karim, A. (2013). “Apparatus for measurement of acoustic wave propagation under uniaxial loading with application to measurement of third-order elastic constants of piezoelectric single crystals,” Rev. Sci. Instrum. 84(5), 054901. 